\title{
Evaluation of an enhanced training package to support clinical trials training in low and middle income countries (LMICs): experiences from the Born Too Soon Optimising Nutrition study
}

Eleanor Jane Mitchell ( $\nabla$ eleanor.mitchell@nottingham.ac.uk)

University of Nottingham School of Medicine https://orcid.org/0000-0002-6998-4533

Jalemba Aluvaala

University of Nairobi

Lucy Bradshaw

University of Nottingham School of Medicine

Jane P Daniels

University of Nottingham School of Medicine

Ashok Kumar

Banaras Hindu University Institute of Medical Sciences

Jacqueline Opira

Kenya Paediatric Research Consortium

Mary Oliver

University of Nottingham School of Education

Phoebe Pallotti

University of Nottingham School of Health Sciences

\section{Zahida Qureshi}

University of Nairobi

Richard Swinden

University of Nottingham School of Medicine

Fredrick Were

University of Nairobi

Shalini Ojha

University of Nottingham School of Medicine

\section{Research Article}

Keywords: clinical trials, training, LMICs, global health, ICH-GCP

Posted Date: December 20th, 2021 
DOl: https://doi.org/10.21203/rs.3.rs-703406/v1

License: (c) (1) This work is licensed under a Creative Commons Attribution 4.0 International License. Read Full License 


\section{Abstract \\ Background}

Training is essential before working on a clinical trial, yet there is limited evidence on effective training methods. In low and middle income countries (LMICs), training of research staff was considered the second highest priority in a global health methodological research priority setting exercise.

\section{Methods}

We explored whether an enhanced training package in a neonatal feasibility study in Kenya and India, utilising elements of the train-the-trainer approach, altered clinicians and researchers' clinical trials knowledge. A lead "trainer" was identified at each site who attended a UK-based introductory course on clinical trials. A two-day in-country training session was conducted at each hospital. Sessions included the study protocol, governance, data collection and ICH-Good Clinical Practice (GCP). To assess effectiveness of the training package, participants completed questionnaires at the start and end of the study period, including demographics, prior research experience, protocol-specific questions, informed consent and ICH-GCP.

\section{Results}

Thirty participants attended in-country training sessions and completed baseline questionnaires. Around three quarters had previously worked on a research study, yet only half had previously received training. Nineteen participants completed questionnaires at the end of the study period. Questionnaire scores were higher at the end of the study period, though not significantly so. Few participants 'passed' the informed consent and ICH-Good Clinical Practice (GCP) modules, using the Global Health Network Training Centre pass mark of $\geq 80 \%$. Participants who reported having prior research experience scored higher in questionnaires before the start of the study period.

\section{Conclusions}

An enhanced training package can improve knowledge of research methods and governance though only small improvements in mean scores between questionnaires completed before and at the end of the study period were seen and were not statistically significant. This is the first report evaluating a clinical trial training package in a neonatal trial in LMICs. Due to the Covid-19 pandemic, research activity was paused and there was a significant time lapse between training and start of the study, which likely impacted upon the scores reported here. Given the burden of disease in LMICs, developing high-quality training materials which utilise a variety of approaches and build research capacity, is critical. 


\section{Background}

Individuals who work on clinical trials should be appropriately qualified and have received training that is relevant and proportionate to their role in accordance with the principles of ICH-Good Clinical Practice (GCP) and national policies and regulations ${ }^{(1,2)}$. However, there is limited methodological research to evaluate clinical trial training methods and programmes both within UK ${ }^{(3)}$ and even more in low-andmiddle income countries (LMICs). In a priority-setting exercise to identify the top priorities for methodological research in trials conducted in LMICs ${ }^{(4)}$, training of research staff was rated the second highest priority. In particular, finding purposeful and cost-effective training methods and using blended learning approaches were highlighted. Only $60 \%$ of the participants of this global survey, who were from LMICs, had received any training. The lack of research capacity is reflected in the outputs for example, Africa bears $25 \%$ of the global burden of diseases, yet produces only $2 \%$ of global research outputs ${ }^{(5)}$.

Groups such as The Global Health Network (www.tghn.org) and many UK-funders of global health research advocate that research projects should include sustainable research capacity-building. Increasing opportunities to provide high-quality training to enhance capacity in LMICs is an essential step towards creating an equitable, global research community.

The "train the trainer" approach where a small group is trained to, in turn, disseminate training to larger groups is known to be an effective way to spread training in the healthcare sectors ${ }^{(6-8)}$. In addition, blended approaches i.e., using a variety of methods, further increase dissemination of curricula ${ }^{(9)}$. We conducted two literature searches in June 2019 and March 2021 and found no studies evaluating such approaches in the context of clinical trials. A study has been developed which utilises a train-the-trainer approach to teaching African-American community leaders in the USA about cancer clinical trials in order to increase public engagement and participation in clinical trials, though is yet to be evaluated ${ }^{(10)}$.

The aim of this study was to explore whether an enhanced training package, which includes elements of the 'train the trainer' approach, altered clinicians and researchers' clinical trials knowledge in clinical researchers involved in a study in LMICs.

\section{Methods}

The "Born Too Soon: Optimising Nutrition" project ran from 1 August 2019 to 31 December 2020, to investigate feeding strategies for preterm infants admitted for neonatal care (reported separately). The project included a prospective observational study, a feasibility randomised controlled trial, and a qualitative study involving healthcare professionals and parents. The study was conducted in two hospitals: The Kenyatta National Hospital, Nairobi, Kenya and the Sir Sunderlal Hospital, Varanasi, India. Both are large teaching hospitals, affiliated with universities. The team in Kenya had prior research

experience $(11,12)(13,14)$ whilst the team in India self-described as having limited experience in conducting research studies, in particular clinical trials. 


\section{Enhanced training package}

The enhanced training package involved several components. Firstly, a designated "trainer" identified from each centre, undertook a 5-day clinical trials' design and conduct course ("Fundamentals of Clinical Trials", Nottingham Clinical Trials Unit: https://www.nctu.ac.uk/short-courses/index.aspx). The trainers (i) were responsible for disseminating training and providing support to other healthcare professionals and researchers at their site; (ii) had access to support and mentorship from experienced researchers based at the project coordinating centre in the UK and (iii) disseminated the study protocol and other documents to all site staff.

Secondly, project leads (EJM and SO) provided face-to-face training session to all site staff in Kenya and India in December 2019. This training consisted of two full-day sessions including training on the study protocol, Good Clinical Practice, research governance, and data collection. Training was delivered via a range of methods including lectures, small group discussions, Q\&A sessions and data entry workshops. Learning was put into practice by mock patient screening and data collection on the Newborn Units. Participants included a range of healthcare professionals (doctors, nurses, midwives) and study personnel (coordinator, data entry personnel).

\section{Evaluation of training package}

Participants were asked for their consent to participate in the study reported here. Study information was provided, and a consent form completed prior to the training session. Participants were asked to complete four paper questionnaires: demographics details; research experience; protocol-specific questions; and questions about informed consent and ICH-GCP. The introduction to Informed consent and ICH-GCP questionnaires were taken from materials publicly available via The Global Health Network training centre (https://globalhealthtrainingcentre.tghn.org/) while the others were developed by the project team (supplementary material 1,2). Baseline questionnaires were returned to the UK and data entered onto a MS Excel ${ }^{\circledR}$ spreadsheet.

At the end of the project, electronic versions (using JISC surveys $\odot$ ) of the protocol-specific questionnaire, ICH-GCP and Introduction to informed consent questionnaires were sent to the participants. The survey opened on 20 November 2020 and closed on 18 December 2020. Two email reminders were sent to encourage completion during this time. Data was downloaded from JISC as a .CSV file.

\section{Evaluation instruments}

The protocol-specific training questionnaire included eight multiple choice response questions, with the highest mark available being 10. The Introduction to informed consent questionnaire (13 items) and the ICH-GCP questionnaire (31 items) both had multiple choice response questions. In accordance with the TGHN training resource, questionnaires were scored as a percentage; a pass mark was $80 \%$ or above.

\section{Statistical analysis}


Data are reported overall and per country. Participant characteristics and previous research experience are summarised descriptively using frequency counts and percentages. The responses to the protocolspecific questionnaire, introduction to informed consent and ICH-GCP questionnaires are summarised descriptively using the mean, standard deviation (SD) and minimum, maximum values; reported overall and per country. For participants who completed questionnaire sets at both time-points, mean differences in scores and $95 \%$ confidence intervals $(\mathrm{Cl})$ are reported. Exploratory analyses were conducted to check for differences in questionnaire scores in individuals who self-reported prior research experience and those who did not. Statistical analyses were undertaken in Stata version 15 (Stata Corp LLC, Texas).

\section{Results}

Thirty participants (17 in Kenya, 13 in India) attended the in-country training sessions in December 2019 including 14 (46\%) nurses and $13(43 \%)$ doctors (Table 1).

\section{Table 1: Participant characteristics}

As shown in Table 2, almost three-quarters had previously worked on a research study $(n=22,73 \%)$ yet only half $(n=11,50 \%)$ had received any research training previously. Most had experience of observational or questionnaire-based studies and only $8 / 22(36 \%)$ had worked on a clinical trial.

\section{Table 2: Participants' previous research experience}

Of the 30 participants who completed the questionnaires at the start of the study, 19 (63\%; 16 in Kenya, 3 in India) completed the questionnaires at the end of the study period. Overall, scores for each questionnaire were higher at the end of the study period, as compared to the scores at the start of the study. The largest differences were seen in questionnaires completed by the team in India (Supplementary Table 1). Only $3(10 \%)$ participants 'passed' (i.e., scored $>80 \%$ ) the consent questionnaire before the start of the study, and 4 (21\%) passed at the end of the study period. No participants 'passed' the ICH-GCP questionnaire before the start of the study and only $1(5 \%)$ passed at the end of the study period.

For the 19 participants who completed both sets of questionnaires, small mean increases were seen in scores at the end of the study period compared to before (Table 3).

Table 3: Differences in questionnaire scores before and at the end of the study period for participants who completed both questionnaire sets

Staff who self-reported as having previous research experience $(n=22)$ scored higher in each questionnaire before the start of the study period, than those who had no prior research experience $(n=8)$ (Table 4). Almost all staff with no prior research experience were in India $(n=7,88 \%)$.

Table 4: Differences in questionnaire scores before start of study period between staff with and without prior research experience 


\section{Discussion}

This study demonstrates that an enhanced training package can improve knowledge of research methods and governance. However, despite some improvement in mean scores, we found that scores did not increase statistically significantly between start and end of the study period.

This study was subjected to an unexpected pause due to the Covid-19 pandemic which limited the sample size, delivery and dissemination of training, and the end of the study assessments. The training leads had attended the 5-day training course in November 2019 and training sessions took place in December 2019. The study was due to commence in February 2020, however there was a delay with receiving ethical approval and once received, the Covid-19 global pandemic had been declared. Clinical staff in the hospitals in Kenya and India understandably had to prioritise clinical activity. The study opened on 1 August 2020, some nine months after training had been undertaken. Whilst the funder granted a small time-only extension, it was not possible to provide complete re-training prior to the start of data collection due to the need to complete the project quickly. Instead, ad-hoc training was provided to the training leads. In addition, the UK study coordinator worked closely with the study coordinators in Kenya and India to answer any queries they had. The long pause in research activity could have substantially contributed to the very small differences seen between questionnaires completed at the start and end of the study period. Despite this, the results are encouraging and provide some evidence to support this model of research training.

The Global Health Network Training centre classifies a 'pass' mark as $\geq 80 \%$ for the introduction to informed consent questionnaire and ICH-GCP questionnaire. In this study the mean scores were substantially lower than this at both time-points but improved by the end of the study (from $56 \%$ and $69 \%$ for informed consent, and from $45 \%$ and $56 \%$ for ICH-GCP). Whilst an increase was seen in scores, particularly in India, scores remained low, indicating that additional training, incorporating alternative methods, with refresher training are required in future studies. The biggest gains in questionnaire scores were in questionnaires completed by participants in India, particularly in the consent questionnaire; with a difference in means of 19 between the beginning and end of the study period. This could perhaps reflect that the team in India were research naïve and had not previously participated in research training of this nature. Only three participants in India completed the questionnaires at the end of the study period; these three participants were very involved in the conduct of the study which could explain the larger increase in scores as they were working on the study on a daily basis, unlike other participants who had a less active role.

A strength of this study is that, to the best of our knowledge, this is the first time a clinical trials training package has been evaluated for use in a global health neonatal trial. However, there are some limitations. The substantial delay between the provision of training and the study starting, imposed by the pandemic, may have affected performance at the end of the study period. We did not assess immediate impact of the training. An additional set of questionnaires at the end of the initial training session could have provided additional information and comparison to assess gain or decline in knowledge over the study 
period. Finally, very few staff $(n=3)$ in India completed the questionnaires at the end of the study period. This could be in part due to the type of staff that attended the original training session and completed baseline questionnaires: the majority were nurses who, by the time the study started, were moved from the project to work clinically for the pandemic response.

Our results demonstrate the need for training for researchers in LMICs, both for individual studies and generically to increase research capacity. This is arguably even more critical since substantial cuts have been made to global health funding by the UK government ${ }^{(15)}$. For research to be conducted where need is greatest, enabling researchers and health workers in LMICs to acquire research and research management skills is essential. Adapting the approach described here, by including additional training sessions throughout the study and providing the "trainers" with more standardised training tools for them to replicate the training when required, could be helpful for the future. During the Covid-19 pandemic, research teams have adapted training materials to enable virtual training sessions, since much travel between countries has been prohibited. Whilst this reduces the cost and environmental impact of inperson training conducted in LMICs by research teams outside of the setting, we strongly believe a blended approach is required. In-person training is important to understand the context and to enable detailed interactions between staff, in order to pick-up on issues that may need further input. Training packages designed to improve the skills of research teams in LMICs should continue to be evaluated, both in order to upskill staff in LMICs and to add to the trials methodology evidence-base. Researchers at the Global Health Network, interested in methodology research, are in the process of developing a core curriculum, via the Essential Research Skills Project

(https://globalresearchmethods.tghn.org/methodology-projects/essential-research-skills-trainingcurriculum/), developed by analysis of training needs surveys, workshops and a Delphi survey, and the content from this curriculum could be utilised to support training in global health studies in the future.

\section{Conclusions}

The enhanced training package utilised in the Born Too Soon: Optimising Nutrition study increased the study team's overall knowledge of the study protocol, informed consent and ICH-GCP. However, differences in questionnaire scores before and at end of the study period were small, and for informed consent and ICH-GCP, lower than the pass mark of $80 \%$ outlined by TGHN. Further research is needed to evaluate clinical trials training packages in LMICs.

\section{Abbreviations}

ICH-GCP

International Conference on Harmonisation-Good Clinical Practice GCP

Good Clinical Practice

LMIC

low and middle income countries 
NCTU

Nottingham Clinical Trials Unit

TGHN

The Global Health Network

$\mathrm{Cl}$

Confidence interval

\section{Declarations}

\section{Ethics approval and consent to participate}

Ethical approval was received by the joint Kenyatta National Hospital-University of Nairobi Ethics Research Committee (ref: P980-12/2019) on 28 February 2020 and the University of Nottingham Faculty of Medical and Health Sciences ethics committee (ref: 433-912) on 25 February 2020.

\section{Consent for publication}

Not applicable

\section{Availability of data and materials}

The datasets used and analysed during the current study are available from the corresponding author upon reasonable request

\section{Competing interests}

No competing interests have been declared.

\section{Funding}

The study was funded by the University of Nottingham Global Challenges Research Fund (GCRF) (ref: RIS 214513).

\section{Authors' contributions}

EJM conceived the idea, designed the study, analysed data and wrote the first draft of the manuscript. MO helped design the study. SO was the Chief Investigator for the overall project. LB provided support with statistical analyses. FW and AK were Principal Investigators at each site, supported by JA and ZQ. In addition, JA and AK were the "trainers" at each site. JO and RS were study coordinators in Kenya and the UK respectively. PP and JD were members of the wider Born Too Soon: Optimising Nutrition team and contributed to ongoing management of the project. All authors helped draft and critique the paper for important intellectual content, and approved the final manuscript.

\section{Acknowledgements}


Many thanks to all staff who participated in the in-country training sessions and who participated in this study by completing questionnaires. Thank you to Joanna Green and Maeve Fitzpatrick, who administer the University of Nottingham Global Challenges Research Fund (GCRF), for their sound advice throughout the project, particularly in relation to 'pause' in activity due to Covid-19. Thank you to the course tutors at the Nottingham Clinical Trials Unit who taught on the 5-day course in November 2019.

\section{References}

1. Health Research Authority. UK Policy Framework for Health and Social Care Research. 2021.

2. European Union Clinical Trials Directive 2001/20/EC. (2001).

3. Townsend D, Mills N, Savović J, Donovan JL. A systematic review of training programmes for recruiters to randomised controlled trials. Trials. 2015;16(1):432.

4. Rosala-Hallas A, Bhangu A, Blazeby J, Bowman L, Clarke M, Lang T, et al. Global health trials methodological research agenda: results from a priority setting exercise. Trials. 2018;19(1):48.

5. Kasprowicz VO, Chopera D, Waddilove KD, Brockman MA, Gilmour J, Hunter E, et al. African-led health research and capacity building- is it working? BMC Public Health. 2020;20(1):1104.

6. Yarber L, Brownson CA, Jacob RR, Baker EA, Jones E, Baumann C, et al. Evaluating a train-the-trainer approach for improving capacity for evidence-based decision making in public health. BMC Health Services Research. 2015;15(1):547.

7. Riley C, Spies LA, Prater L, Garner SL. Improving Neonatal Outcomes Through Global Professional Development. Advances in neonatal care: official journal of the National Association of Neonatal Nurses. 2019;19(1):56-64.

8. de Beurs DP, de Groot MH, de Keijser J, Mokkenstorm J, van Duijn E, de Winter RF, et al. The effect of an e-learning supported Train-the-Trainer programme on implementation of suicide guidelines in mental health care. J Affect Disord. 2015;175:446-53.

9. Pearce J, Mann MK, Jones C, van Buschbach S, Olff M, Bisson JI. The Most Effective Way of Delivering a Train-the-Trainers Program: A Systematic Review. Journal of Continuing Education in the Health Professions. 2012;32(3):215-26.

10. Blakeney N, Michaels M, Green M, Richmond A, Long D, Robinson WS, et al. Collaborative development of clinical trials education programs for African-American community-based organizations. Journal of cancer education: the official journal of the American Association for Cancer Education. 2015;30(2):400-6.

11. Mitchell EJ, Benjamin S, Ononge S, Ditai J, Qureshi Z, Masood SN, et al. Identifying women giving birth preterm and care at the time of birth: a prospective audit of births at six hospitals in India, Kenya, Pakistan and Uganda. BMC Pregnancy Childbirth. 2020;20(1):439.

12. Mitchell EJ, Qureshi ZP, Were F, Daniels J, Gwako G, Osoti A, et al. Feasibility of using an Early Warning Score for preterm or low birthweight infants in a low-resource setting: results of a mixedmethods study at a national referral hospital in Kenya. BMJ Open. 2020;10(10):e039061. 
13. Woman Trial Collaborators. Effect of early tranexamic acid administration on mortality, hysterectomy, and other morbidities in women with post-partum haemorrhage (WOMAN): an international, randomised, double-blind, placebo-controlled trial. Lancet. 2017;389(10084):2105-16.

14. Widmer M, Piaggio G, Nguyen TMH, Osoti A, Owa OO, Misra S, et al. Heat-Stable Carbetocin versus Oxytocin to Prevent Hemorrhage after Vaginal Birth. N Engl J Med. 2018;379(8):743-52.

15. Sample I, Editor S. UK scientists warn of catastrophic impact of funding cuts. The Guardian. 202131 March 2021. https://www.theguardian.com/science/2021/mar/31/uk-scientists-funding-cuts-grantsforeign-aid [accessed 5 April 2021].

\section{Tables}

Table 1: Participant demographics

\begin{tabular}{|c|c|c|c|}
\hline & $\begin{array}{c}\text { Overall } \\
\mathrm{n}=30(\%)\end{array}$ & $\begin{array}{c}\text { Kenya } \\
\mathrm{n}=17(\%)\end{array}$ & $\begin{array}{c}\text { India } \\
\mathrm{n}=13(\%)\end{array}$ \\
\hline \multicolumn{4}{|l|}{ Gender } \\
\hline Male & $7(23)$ & $4(24)$ & $3(23)$ \\
\hline Female & $23(77)$ & $13(76)$ & $10(77)$ \\
\hline \multicolumn{4}{|l|}{ Age } \\
\hline $16-25$ & $3(10)$ & $1(6)$ & $2(15)$ \\
\hline $26-34$ & $8(27)$ & $1(6)$ & $7(54)$ \\
\hline $35-44$ & $9(30)$ & $7(41)$ & $2(15)$ \\
\hline $45-54$ & $2(7)$ & $2(12)$ & $0(0)$ \\
\hline $55+$ & $7(23)$ & $6(35)$ & $1(8)$ \\
\hline missing & $1(3)$ & $0(0)$ & $1(8)$ \\
\hline \multicolumn{4}{|l|}{ Job Title } \\
\hline Professor & $3(10)$ & $2(12)$ & $1(8)$ \\
\hline Paediatrician & $2(7)$ & $2(12)$ & $0(0)$ \\
\hline Consultant & $1(3)$ & $1(6)$ & $0(0)$ \\
\hline Nurse & $10(33)$ & $4(34)$ & $6(46)$ \\
\hline Associate Professor & $2(7)$ & $1(6)$ & $1(8)$ \\
\hline Study coordinator & $1(3)$ & $1(6)$ & $0(0)$ \\
\hline Data clerk & $1(3)$ & $1(6)$ & $0(0)$ \\
\hline Nurse manager/chief nurse/coordinator & $4(13)$ & $3(18)$ & $1(8)$ \\
\hline Lecturer & $1(3)$ & $1(6)$ & $0(0)$ \\
\hline Social scientist & $1(3)$ & $1(6)$ & $0(0)$ \\
\hline Junior (resident) doctor & $4(13)$ & $0(0)$ & $4(31)$ \\
\hline \multicolumn{4}{|l|}{ Clinical area } \\
\hline Obstetrics & $1(3)$ & $1(6)$ & $0(0)$ \\
\hline Neonatology & $21(70)$ & $9(53)$ & $12(92)$ \\
\hline Midwifery & $2(7)$ & $2(12)$ & $0(0)$ \\
\hline General nursing & $2(7)$ & $2(12)$ & $0(0)$ \\
\hline NA & $3(10)$ & $3(18)$ & $0(0)$ \\
\hline Missing & $1(3)$ & $0(0)$ & $1(8)$ \\
\hline
\end{tabular}

Table 2: Previous research experience 


\begin{tabular}{|c|c|}
\hline & $\mathrm{n}=30(\%)$ \\
\hline \multicolumn{2}{|l|}{ Previously worked on a research study before } \\
\hline 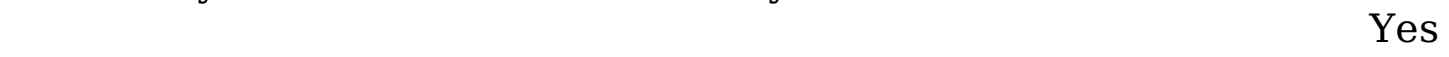 & $22(73)$ \\
\hline No & $8(27)$ \\
\hline & $\mathrm{n}=22(\%)$ \\
\hline \multicolumn{2}{|l|}{ Number of research studies previously worked on } \\
\hline ( & $12(55)$ \\
\hline 4-6 & $2(9)$ \\
\hline $7-10$ & $1(4)$ \\
\hline $10+$ & $6(28)$ \\
\hline Don't remember & $1(4)$ \\
\hline \multicolumn{2}{|l|}{ Last time worked on a research study } \\
\hline Currently & $15(68)$ \\
\hline In the last year & $3(14)$ \\
\hline More than a year ago & $4(19)$ \\
\hline \multicolumn{2}{|l|}{ Types of studies previously worked on - all that applied ticked } \\
\hline Observational (no intervention) & $15(68)$ \\
\hline Clinical trial & $8(36)$ \\
\hline Questionnaire-based study & $15(68)$ \\
\hline Case studies & $1(5)$ \\
\hline Don't know & $1(5)$ \\
\hline Qualitative & $2(10)$ \\
\hline Systematic review & $1(5)$ \\
\hline \multicolumn{2}{|l|}{ Previously received research training } \\
\hline 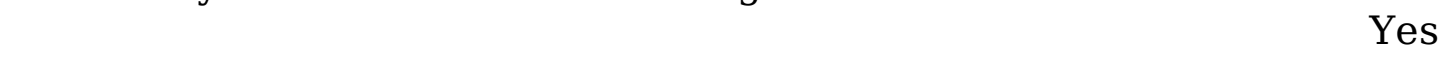 & $11(50)$ \\
\hline No & $10(45)$ \\
\hline $\begin{array}{lll}2 & \text { Missing }\end{array}$ & $1(5)$ \\
\hline \multicolumn{2}{|l|}{$\begin{array}{r}\text { Tasks undertaken in previous research studies - all that applied ticked } \\
\text { Overall study responsibility as Principal Investigator }\end{array}$} \\
\hline Study coordination / management & 10 \\
\hline Obtaining consent from participants & 10 \\
\hline Recruiting participants & 11 \\
\hline Data collection & 10 \\
\hline Data entry & 12 \\
\hline Data analysis & 11 \\
\hline Reporting & 8 \\
\hline & 10 \\
\hline
\end{tabular}

Table 3 - Differences in questionnaire scores before and at the end of the study period for participants who completed both questionnaire sets 


\begin{tabular}{|r|c|c|c|}
\hline & $\begin{array}{c}\text { Before } \\
(\mathrm{n}=19)\end{array}$ & $\begin{array}{c}\text { After } \\
(\mathrm{n}=19)\end{array}$ & Difference in means (SD), 95\% CI \\
\hline Protocol-specific & $5.6(1.8)$ & $5.8(2.6)$ & $\begin{array}{l}0.2(2.1), \\
-0.8 \text { to } 1.2\end{array}$ \\
\hline $\begin{array}{r}\text { Mntroduction to informed consent } \\
\text { Mean score (SD) }\end{array}$ & $64(17)$ & $69(14)$ & $\begin{array}{c}5(12), \\
0.6 \text { to } 11.1\end{array}$ \\
\hline $\begin{array}{c}\text { ICH-Good Clinical Practice } \\
\text { Mean score }\end{array}$ & $53(9.4)$ & $56(14)$ & $\begin{array}{l}3.2(12.7), \\
-2.9 \text { to } 9.3\end{array}$ \\
\hline
\end{tabular}

Table 4: Differences in questionnaire scores before start of study period between staff with and without prior research experience

\begin{tabular}{|c|c|c|}
\hline & $\begin{array}{c}\text { Staff with prior research } \\
\text { experience }^{1}\end{array}$ & $\begin{array}{l}\text { Staff without prior research } \\
\text { experience }^{1}\end{array}$ \\
\hline & $\mathrm{n}=22$ & $\mathrm{n}=8$ \\
\hline $\begin{array}{r}\text { Protocol-specific } \\
\text { Mean score (SD) } \\
\text { min, max }\end{array}$ & $\begin{array}{c}6.0(1.6) \\
3,8\end{array}$ & $\begin{array}{c}3.4(1.9) \\
2,6\end{array}$ \\
\hline $\begin{array}{l}\text { Introduced to informed } \\
\text { consent } \\
\text { Mean score (SD) } \\
\text { min, max }\end{array}$ & $\begin{array}{l}62(18) \\
23,85\end{array}$ & $\begin{array}{l}41(10) \\
30,62\end{array}$ \\
\hline $\begin{array}{l}\text { ICH-Good Clinical } \\
\text { Practice } \\
\qquad \begin{array}{r}\text { Mean score (SD) } \\
\text { min, max }\end{array}\end{array}$ & $\begin{array}{c}50(13) \\
11,67\end{array}$ & $\begin{array}{l}31(12) \\
17,50\end{array}$ \\
\hline
\end{tabular}

${ }^{1}$ Self-reported at time of completion of previous research experience questionnaire

\section{Supplementary Files}

This is a list of supplementary files associated with this preprint. Click to download.

- DemographicsQuestionnaireSupplementaryMaterial1.docx

- ProtocolquestionnaireSupplementaryMaterial2.docx

- SupplementaryTable1ForSubmission6Jul21.docx 